\title{
Evidence Disclosure and Severity of Punishments
}

\author{
Evsen Turkay *
}

October 31, 2010

\begin{abstract}
The relationship between legal offenses and punishment is well studied by scholars of sociology, economics and law. Economists contend that punishment is a cost of committing an offense, hence an increase in the severity of punishments should decrease incentives to commit legal offenses. And the efficiency of legal punishments are studied generally from this perspective: giving efficient incentives to commit legal offense. This paper studies the relationship between punishment and evidence disclosure. A defendant is trying to persuade a judge by presenting evidence to take a favorable legal action rather than less favorable ones on his case. I show that the equilibrium disclosure of the defendant is not affected by a change in the scale of legal actions when there is no uncertainty on how the judge evaluates evidence. With uncertainty, however, the defendant can be induced to disclose more information by decreasing the severity ratio of the most unfavorable legal action to the most favorable one. This shows that in the more realistic case of uncertainty the severity of punishments has an effect on evidence disclosure and efficiency of punishment schedule should be analyzed by internalizing its effect on evidence disclosure as well.
\end{abstract}

\section{Introduction}

Severity of punishments has been analyzed from the angle of efficiency by several scholars and he extent of the effectiveness of legal punishments in deterring legal offenses have been exten-

*I am grateful to my advisors Jan Werner and Marcel Richter for their guidance and advice in every step of my project, and also for their constant encouragement. I am also grateful to Itai Sher for our numerous discussions and his invaluable advice on my paper. I am thankful to Beth Allen, David Rahman, Aldo Rustichini, Fatih Guvenen who were always willing to listen and gave me useful suggestions. Unni Pillai, Simge Tarhan, Ayca Ozdogan, Gina Pieters, Ilenin Kondo and Ioanna Gypari helped me in various stages of this research with their valuable feedback. I am thankful to participants of Mathematical Economics seminar in University of Minnesota. I am also thankful to participants of SIDE-ISLE 2009 and especially Simona Benedettini for detailed comments and suggestions. This version is preliminary and incomplete. All errors in the paper are my own. 
sively studied and debated. ${ }^{1}$ In his seminal paper, Becker (1968) analyzed optimality of legal punishments using a model in which the offenders are rational decision makers who perceives legal punishments as a cost of crime and decides on whether to commit crime accordingly. Recent papers like Dezhbakhsh, Rubin, and Shepherd (2003), Mocan and Gittings (2003) and Donohue and Wolfers (2009) studied the effect of capital punishment on murders and hence contributed to the discussion on the effect of severity on legal offenses. In contrast to the focus of previous literature on legal punishments, my paper studies the relationship between punishment and evidence disclosure.

Evidence production is crucial for accuracy of the legal process as Posner (1999) contends. To see the importance of evidence production consider an increase in the amount of evidence produced during trials. This would lead to an increase in the accuracy of the legal procedure which in turn would increase the confidence in the legal system and hence affect incentives to commit crime. Hence, to have a complete analysis of optimality of punishments, punishments should also be analyzed in relation to evidence disclosures. Evidence disclosures are crucial for courts to make informed legal decisions. How much evidence a defendant discloses in court depends on what results these disclosures may lead to, which in turn depends on the severity of punishments. This paper studies the relationship between the severity of legal punishments and evidence disclosed in courts. The model proposed in this paper is general enough to address different kinds of cases like cases in criminal, tort or contract law, I use the example of a tort case to illustrate the specifics and the results of the model.

Consider the example of a judge trying to decide on the punishment for a defendant in a tort case. The defendant has a piece of evidence that the defendant can either disclose it or suppress it but cannot modify it in any other sense as in Bull and Watson (2007) and Bull and Watson (2004). I restrict my attention to the cases in which the sole provider of information about the evidence is the defendant; neither judge nor any other agent can investigate the matter themselves and find evidence. As discussed in Shin (1994), the possibility that the defendant is uninformed leads the judge to make non-trivial inferences when the defendant does not answer a question or suppress information. I focus on the relationship between the defendants' decision and the severity of legal actions he is facing. To solidify the abstract situation even further, assume that the defendant is a producer whose product has harmed a consumer. The judge determines the extent of compensation according to whether the producer has been irresponsible or not, among other factors. If the product was tested for a specific characteristic before it was sent into the market, the producer may choose to present the test results. The judge might choose a small

\footnotetext{
${ }^{1}$ For a survey of empirical papers that support the argument that punishment is indeed a deterrent to criminal activity, see Tullock (1974).
} 
compensation payment upon seeing favorable test results or might choose a high compensation amount if the test results show that the producer was irresponsible. The producer may not be certain how the evidence he presents would affect his case, so the producer weights the risk of being perceived as irresponsible against the possibility of being perceived as an unlucky person who made the best possible decision under the light of what he knows. The severity of legal punishments would surely affect the decision of the producer.

Throughout the paper I focus on the case that the defendant faces some uncertainty about how the evidence would affect the decision on his case. There are two reasons for this. First, judicial decision making bodies, the judge hereon, exhibit a wide variety of point of views when it comes to interpreting laws, due to their private knowledge, previous experiences, political views etc. Hence assuming that the defendant faces uncertainty about how the evidence is going to affect his case is a realistic assumption. Secondly, the case that the defendant knows the effect of the evidence on his case with certainty is a special case of persuasion games that has been analyzed thoroughly in the literature in Milgrom (1981), Dye (1986), Shin (1994), Glazer and Rubinstein (2004), Glazer and Rubinstein (2006), Sher (2008). These papers analyzed similar games of communication and it is clear that beneficial evidence is going to be presented, whereas harmful evidence is going to be suppressed. This paper contributes to the literature of evidence disclosure and persuasion games in two ways. First, I extend the model in Che and Severinov (2007) so that evidence can have a positive, negative or negligible effect on the case for the defendant rather than having a binary effect of punishing or acquitting the defendant. Enriching the set of legal consequences the evidence might have enables me to propose a definition of severity of legal consequences. Second, I compare the effect of a change in the scale of legal actions on the disclosures of the defendant when there is uncertainty about the judge's type and when there is no uncertainty; uncertainty proves to be an important characteristic that determines whether severity of punishments would or would not have any affect on the disclosures of the defendant. Following the tort case example in which the schedule of legal punishments is in the form of a monetary compensation that the defendant has to pay. I show that:

1. The equilibrium disclosure of the producer is not affected by a change in the compensation schedule when there is no uncertainty on the type of the listener (judge).

2. With uncertainty, however, the defendant can be induced to disclose more information by decreasing the severity of compensation schedule.

3. The defendant would disclose less information when the severity of compensation schedule is increased.

This implies that uncertainty leads to a negative relationship between the disclosures of the 
defendant and the severity of legal actions. In cases where the producer knows how the evidence will be perceived by the judge, a change in the compensation schedule does not affect what evidence he suppresses and what evidence he discloses. The severity of the compensation schedule would affect the producer's evidence disclosure behavior only if the producer faced uncertainty about how the judge evaluates the evidence. In general, in a legal system in which there is no uncertainty and every contingency is well-defined by laws, a change in the size legal actions does not affect incentives to disclose evidence. However, in a legal system where the laws leave a higher degree of freedom of choice to the judge, i.e. when there is uncertainty about the listener's type, decreasing the severity of unfavorable legal action relative to the favorable one would affect evidence disclosed in equilibrium, positively.

\section{Evidence Disclosure Game}

A defendant (he) is facing a judge (she) in a legal proceeding. The judge chooses an action that determines the utility of the defendant. The defendant sends a message $m$ to the judge in order to persuade the judge to take a favorable legal action. The defendant faces uncertainty about how the judge would evaluate the evidence. Turning back to the example of the tort case presented in the introduction, a lenient judge might decide to reduce the defendant's punishment when mediocre results are presented to court while a strict judge might increase the punishment of the defendant for the same test results.

There are two pieces of information related to judge's preferred action for the case. The first piece of information is about the nature of evidence, denoted by $x$. The second piece of information is the type of the judge and that determines how she (the judge) evaluates what is presented to her. The evidence is hard in the sense that if the defendant has evidence, then he can either disclose the evidence or hide it, but cannot forge or manipulate the evidence. In the tort case example, the producer who tested their product can show the results to the court or can hide them, but forging fake results is extremely costly.

The defendant might be informed or uninformed in the sense that he may or may not have evidence. The informed defendant observes evidence $x$ drawn from the interval $[0,1]$ in the beginning of the game. The uninformed defendant's type is denoted with $x_{o}$ and if the defendant is uninformed he does not observe any evidence, he is a producer who has not tested his product before sending it to the market. The type space for the defendant is $\Theta=[0,1] \cup\left\{x_{o}\right\}$. The prior probability on $\Theta$ is atomless on $[0,1]$ and $Q\left(x_{o}\right)>0$. Modeling the uninformed type $x_{o}$ as a separate point in the type space enables me to model the behavior of a judge who wants to base her decision solely on the available evidence if there exists some evidence and who wants to decide on bare-bones facts of the case if there exists no evidence. This way of modeling is in contrast to the judge described in Che and Severinov (2007). In Che and Severinov's model the 
judge decides which action to take based on her belief about the state of the nature whether the defendant is informed or uninformed.

The type of the defendant determines what he can say in the court- in other words, which messages are available to him. The uninformed defendant's message set consists of one message $m_{o}$. The informed defendant who observes evidence $x$ has the message set $M_{x}=\left\{m_{x}, m_{o}\right\}$. Message $m_{o}$ is available to all types of the defendant and peculiar to none. This characteristic of $m_{o}$ leads it to be interpreted as suppressing evidence, if it is sent by an informed type. The set of messages available in the evidence disclosure game is $\mathbb{M}=[0,1] \cup\left\{m_{o}\right\}$. This message structure is widely used in disclosure games with verifiable information as seen in Milgrom (1981), Dye (1986), Shin (1998) and Che and Severinov (2007) ${ }^{2}$. The judge evaluates the evidence according to her private information which is her type $j \in \mathbb{J}$. The variable $j$ represents the judge's interpretation of how the evidence fits in the case given the judge's personal character and his private knowledge about the case. I assume that there is a finite number of types for the judge and denote the set of types with $\mathbb{J}$.

When the defendant sends $m_{o}$, the judge forms beliefs regarding whether the defendant is suppressing evidence or he is genuinely uninformed. The set of judge's actions $\mathbb{A}=\{U, N, F\}$ is a list of legal actions that the judge can impose on the defendant. In response to the disclosure of the defendant, the judge may choose between three actions: Unfavorable (U), Neutral (N) and Favorable (F). For the tort case example these three actions can be interpreted as reducing the compensation that the defendant has to pay, not changing it, or increasing the compensation.

The defendant's utility depends on the action the judge chooses but not on his type, and his utility is described by the function $v: \mathbb{A} \rightarrow \mathbb{R}$ such that $v(F)>v(N)>v(U)$. The defendant chooses his disclosure strategy to get a favorable decision from the judge. Without loss of generality, let $v(N)=0$.

The judge's utility depends on the action she chooses, the type of the defendant, and the type of the judge. As in Daughety and Reinganum (2000) and Che and Severinov (2007), the judge chooses her action according to given legal standards. The legal standard is described by three values: $b_{F}, b_{N}, b_{U}$ such that $b_{F}>b_{N}>b_{U}$. For any pair $(j, \theta)$, the judge evaluates the case by a function $g: \mathbb{J} \times \Theta \rightarrow\left[b_{U}, b_{F}\right]$ and chooses an action. The utility function of the judge $u: \mathbb{J} \times \mathbb{A} \times \Theta \rightarrow \mathbb{R}$ is given by a squared loss function similar to Shin (1998)

$$
u_{j}(a, \theta)=-\left(g(j, \theta)-b_{a}\right)^{2}
$$

\footnotetext{
${ }^{2}$ This implies that the evidence is "hard" in the sense that the defendant cannot modify the evidence. Bull and Watson (2004) discusses implementability in settings with hard evidence. Message $m_{o}$ corresponds to what "cheap document" refers in Bull and Watson (2004) and message $m_{x}$ corresponds to "positive evidence" of state $x$.
} 
Without loss of generality, I assume that $b_{N}=0$. The legal standards may be interpreted as a standard measure of the degree of the guilt of the defendant with which the judge compares her private evaluation of the case. The judge's utility is negatively related to the difference between her evaluation and the legal standards that correspond to the action she chose. If the evaluation is closer to $b_{F}$ than $b_{N}$ or $b_{U}$, then she decides the most suitable action is $F$; whereas a value closer to some other $b_{a}$ implies that she chooses $a$. Upon a tie between two actions, the judge may choose either action and may choose to randomize between two actions.

The evaluation function satisfies two properties for all types of the judge.

Assumption 1. For all $j$ the evaluation function $g(j, x)$ is continuous and monotone increasing in $x \in[0,1]$.

Similar to Che and Severinov (2007), Assumption 1 implies that the higher $x$ is more favorable for the defendant regardless of the type of the judge. But different types of judge may differ on their evaluations for a specific $x^{3}$. For example, a strict type of judge may decide to take action $N$ upon receiving evidence $x$ but a more lenient type may decide to take action $F$.

Assumption 2. For all $j$ the evaluation function $g(j, x)$ satisfies

$$
g(j, 1)=b_{F} \text { and } g(j, 0)=b_{U}
$$

Assumption 2 states that all types of judge agree on how to evaluate the highest and lowest evidence in the sense that all types wants to take action $F$ when $x=1$ and $U$ when $x=0$.

Assumption 3. For all $j, g\left(j, x_{o}\right)=0$.

Assumption 3 means that in the absence of new evidence, the judge takes a neutral action and also implies that the decision of the judge depends solely on the available evidence rather than what the state of the nature may be.

The evidence disclosure game is denoted by $\Gamma(\Theta, P, \mathbb{A})$ when the type of the judge is not observable and with $\Gamma(\Theta, j, \mathbb{A})$ when the type of the judge is known to be $j$. The defendant's strategy is a function that assigns a probability distribution on messages to each type of the defendant, $d: \Theta \rightarrow \mathbb{M}, d(x) \in \Delta M_{x}$. The decision strategy of judge of type $j$ is a function that gives a probability distribution over the set of actions for each message, $f_{j}: \mathbb{M} \rightarrow \Delta \mathbb{A}$.

Upon receiving a message $m$, the judge forms beliefs about the type of the defendant and chooses an action accordingly. The action of the judge depends on the expected utility calculation

\footnotetext{
${ }^{3}$ See Seidmann (2005) for an evidence disclosure model in which defendant's evidence is not ranked the same by different types of the decision maker.
} 
of the judge given her beliefs about the type of the defendant. Upon receiving a message $m$, the judge is said to be best responding given beliefs $\mu$ if the action $a^{*}$ she chooses solves the problem

$$
\max _{a} E_{\mu}\left(-\left(g(j, \theta)-b_{a}\right)^{2} \mid m\right)
$$

That is, $a^{*}$ is a maximizing choice for $j$ if and only if

$$
-\int_{\Theta}\left(g(j, \theta)-b_{a^{*}}\right)^{2} d \mu(\theta \mid m) \geq-\int_{\Theta}\left(g(j, \theta)-b_{a}\right)^{2} d \mu(\theta \mid m)
$$

for all $a$. This is equivalent to

$$
\int_{\Theta}\left[2 g(j, \theta) b_{a^{*}}-b_{a^{*}}^{2}\right] d \mu(\theta \mid m) \geq \int_{\Theta}\left[2 g(j, \theta) b_{a}-b_{a}^{2}\right] d \mu(\theta \mid m)
$$

Rearranging the terms yields

$$
E_{\mu}\left(g(j, \theta) \mid m_{o}\right)\left(b_{a^{*}}-b_{a}\right) \geq \frac{b_{a^{*}}^{2}-b_{a}^{2}}{2}
$$

For $b_{a^{*}}>b_{a}$, this is equivalent to

$$
E_{\mu}(g(j, \theta) \mid m) \geq \frac{b_{a^{*}}+b_{a}}{2}
$$

and for $b_{a^{*}}<b_{a}$

$$
E_{\mu}(g(j, \theta) \mid m) \leq \frac{b_{a^{*}}+b_{a}}{2}
$$

Inequalities 3 and 4 yield cutoffs that the judge compares her evaluation with, and chooses an action accordingly. Since $b_{N}=0$, it follows 3 and 4 from $a^{*}=H$ if and only if

$$
b_{F} \geq E_{\mu}(g(j, \theta) \mid m) \geq \frac{b_{F}}{2}
$$

And similarly, $a^{*}=N$ if and only if,

$$
\frac{b_{F}}{2} \geq E_{\mu}(g(j, \theta) \mid m) \geq \frac{b_{U}}{2}
$$

I introduce the following cutoffs

$$
c_{N}=\frac{b_{U}}{2} \text { and } c_{F}=\frac{b_{F}}{2}
$$

When the judge's evaluation is less than $c_{N}$, she takes action $U$; when it is in between two cutoffs, she takes action $N$; and when the evaluation is greater than the second threshold, she takes action $F$. Let $c_{a_{+}}$denote the cutoff that is higher than and adjacent to $c_{a}$ and for completeness of notation let $c_{U}=b_{U}$ and $c_{F_{+}}=b_{F}$. Note that since $b_{N}=0$, the right hand sides of (3) and (4) can be rewritten as $c_{F}$ and $c_{N}$. I conclude that when message $m$ is received, the judge's best response is $a^{*}$ if and only if

$$
c_{a_{+}^{*}} \geq E_{\mu}(g(j, \theta) \mid m) \geq c_{a^{*}}
$$




\section{Equilibrium Analysis}

I employ an equilibrium notion under which the judge makes a Bayesian inference upon receiving $m_{o}$ and the beliefs of the judge is derived from the equilibrium strategy of the defendant ${ }^{4}$.

Definition. A Sequential Equilibrium of disclosure game is a tuple consisting of the judge's strategy $\left\{f_{1}^{*}, . ., f_{J}^{*}\right\}$, the defendant's strategy $d^{*}$, and beliefs of the judge $\mu$ such that:

1. The defendant's strategy $d^{*}$ is a best response to $f^{*}$. That is, if $d^{*}(x)=m$, then

$$
\sum_{a} \sum_{j} v(a) f_{j}^{*}(a \mid m) P(j)=\max _{\hat{m} \in\left\{x, m_{o}\right\}} \sum_{a} \sum_{j} v(a) f_{j}^{*}(a \mid \hat{m}) P(j)
$$

for all $x$.

2. If $f_{j}^{*}(a \mid m)>0$, then $a$ is a best response for beliefs $\mu$, i.e. $a$ solves (1).

3. For every Borel subset $X$ of $\Theta, \mu\left(\theta \in X \mid m=m_{o}\right)$ is derived from $d^{*}$ via Bayes' rule and for every $x \in[0,1], \mu(\theta=x \mid m=x)=1$.

The question I ask is how does a change in the set of punishments effect evidence disclosures in equilibrium, that is the effect on the set of equilibrium disclosures. I show that if the defendant knows the effect of the evidence he presents on the judge's verdict. In the tort example, this means that if the producer knows $j$, then a change in the compensation schedule does not affect his disclosure behavior. However if the defendant is not sure which evidence invokes $U$, which invokes $N$ and which one invokes $F$, then the changes in possible legal actions do affect the disclosure behavior of the defendant.

I first show the existence of SE. I focus on a particular plausible kind of equilibrium in which the defendant's strategies are in the form of threshold strategies.

Definition. The speaker strategy $d$ is called a threshold strategy if there exists a threshold $\underline{x}$

$$
\underline{x} \geq x \text { implies } d\left(m_{o} \mid x\right)=1 \text {, and } x>\underline{x} \text { implies } d(m=x \mid \theta=x)=1
$$

\footnotetext{
${ }^{4}$ In the United States, defendants are protected from self incrimination by the right to silence. The effects of this protection is frequently debated in the literature and some scholars contend that the right to silence leads to a separating equilibrium, see Seidmann and Stein (2000). In most cases the jury members are advised not to make negative inferences when a defendant uses that right. However, Criminal Justice and Public Order Act 1994 of the UK allows adverse inferences to be drawn from silence. Also in the United States many studies show that contrary to instructions given, jury members make inferences upon silence in reality. For example, the National Legal Poll (1998) of National Law Association indicated that $\% 40$ of the potential jurors would draw a negative inference from the defendants refusal to testify
} 
Theorem 1 Under $A 1-2$, there exists a SE $\left\{f_{1}^{*}, . ., f_{J}^{*}, d^{*}, \mu\right\}$ such that $d^{*}$ is a threshold strategy.

Proof. Assume that the defendant uses a threshold disclosure strategy $d^{*}$ with threshold $\underline{x}$. The beliefs $\mu$ of the judge that are derived this strategy via Bayes' rule are such that

$$
\mu\left(x_{o} \mid m_{o}\right)=\frac{Q\left(x_{o}\right)}{Q\left(x_{o}\right)+Q[0, \underline{x}]}
$$

and,

$$
\mu\left(X \mid m_{o}\right)=\frac{\int_{X} d Q(x)}{Q\left(x_{o}\right)+Q[0, \underline{x}]}
$$

for every Borel subset $X$ of $[0, \underline{x}]$.

Upon receiving $m_{o}$, the expected evaluation of the judge given belief $\mu$ of (8) and (9) is

$$
E_{\mu}\left(g(j, \theta) \mid m_{o}\right)=\int_{\Theta} g(j, \theta) d \mu\left(\theta \mid m_{o}\right)
$$

Using (9) and $g\left(j, x_{o}\right)=0$, expression (10) is equal to

$$
\frac{Q\left(x_{o}\right) g\left(j, x_{o}\right)+\int_{0}^{\underline{x}} g(j, x) d Q(x)}{Q\left(x_{o}\right)+Q[0, \underline{x}]}
$$

Let conv $S$ denote the convex hull of a set $S$. Consider a correspondence $\Psi_{j}:\left[b_{L}, b_{H}\right] \Rightarrow \mathbb{R}$

$$
\Psi(k):=\operatorname{conv}\left\{v(a) \mid c_{a_{+}} \geq k \geq c_{a}\right\}
$$

Correspondence $\Psi(k)$ gives the possible set of utilities of the defendant when the judge's evaluation of the evidence is equal to $k$. The correspondence $\Psi$ is convex, compact valued, and upper-hemicontinuous. In order to show that there exists a threshold equilibrium, it suffices to show that there exists $\underline{x}^{*}$ such that a defendant who observes $\underline{x}^{*}$ is indifferent between disclosing it or sending $m_{o}$ if the judge uses (6) to choose an action. If the defendant is indifferent between disclosing the evidence and sending $m_{o}$ at $\underline{x}^{*}$, then for any $x>\underline{x}^{*}$, disclosing is at least as good as sending $m_{o}$ and for any $x<\underline{x}^{*}$ sending $m_{o}$ is at least as good as disclosing the evidence.

Consider the correspondence

$$
h(\underline{x})=\sum_{j} \Psi(g(j, \underline{x})) P(j)-\sum_{j} \Psi\left(\frac{Q\left(x_{o}\right) g\left(j, x_{o}\right)+\int_{0}^{\underline{x}} g(j, x) d Q(x)}{Q\left(x_{o}\right)+Q[0, \underline{x}]}\right) P(j)
$$

The first term on the right hand side of (13) is the set of possible expected utilities of the defendant when the defendant discloses $\underline{x}$ and the judge gives a best response to the defendant. The second term on the RHS is the set of expected utilities of the defendant when he sends $m_{o}$ 
and the judge's evaluation is given by (10) where $\mu$ is defined by (9) for threshold $\underline{x}^{*}$. If there exists $\underline{x}^{*} \in[0,1]$ such that $0 \in h\left(\underline{x}^{*}\right)$, then

$$
\sum_{j} \Psi\left(g\left(j, \underline{x}^{*}\right)\right) P(j)=\sum_{j} \Psi\left(\frac{Q\left(x_{o}\right) g\left(j, x_{o}\right)+\int_{0}^{\underline{x}^{*}} g(j, x) d Q(x)}{Q\left(x_{o}\right)+Q\left[0, \underline{x}^{*}\right]}\right) P(j)
$$

Such $\underline{x}^{*}$ is a threshold above which the defendant prefers to disclose the evidence and under which the defendant prefers to send $m_{o}$ while the judge is giving a best response to the disclosure strategy with a threshold $\underline{x}^{*}$. I want to show such $\underline{x}^{*}$ exists. Function $g(j, x)$ is continuous in $x \in[0,1]$ and $h$ is an upper-hemicontinuous, compact and convex valued correspondence. By Assumption $2, h(1) \geq 0$ and $h(0) \leq 0$. Therefore by the Intermediate Value Theorem for correspondences, see de Clippel (2008), there exists $\underline{x}^{*}$ such that $0 \in h\left(\underline{x}^{*}\right)$. Therefore,

$$
0 \in \sum_{j} \Psi\left(g\left(j, \underline{x}^{*}\right)\right) P(j)-\sum_{j} \Psi\left(\frac{Q\left(x_{o}\right) g\left(j, x_{o}\right)+\int_{0}^{\underline{x}^{*}} g(j, x) d Q(x)}{Q\left(x_{o}\right)+Q\left[0, \underline{x}^{*}\right]}\right) P(j)
$$

This implies that $\underline{x}^{*}$ is a threshold such that if the defendant observes $\underline{x}^{*}$, then he is indifferent between disclosing the evidence or sending $m_{o}$, so (14) characterizes a threshold equilibrium.

I analyze the effects of a change in the set of actions available to the judge, for example an increase in the legal punishments, on the disclosure policy the defendant will choose in equilibrium. The utility function of the judge implies that the only objective of the judge is to choose the the action that complies with exogenously defined legal standards $\left\{b_{a}\right\}_{a \in \mathbb{A}}$. This restriction on the judge's utility implies that a change in the severity of legal actions does not have an effect on the utility of the judge.

I focus on the changes in the least favorable action for the defendant relative to the most favorable action that the judge can give for any given set of actions. Therefore, I restrict alternative action sets to such sets that the neutral action of the judge yields zero utility to the defendant. ${ }^{5}$ Let $\mathbf{A}=\left\{\mathbb{A}^{\prime}\right.$ such that $\left.\left|A^{\prime}\right|=3, N^{\prime}=N\right\}$ be the collection of such alternative action sets where $N$ is kept constant. The action sets in $\mathbf{A}$ should be interpreted as deviations from $\mathbb{A}$ in terms of severity. The ratio $r_{A}=|v(U)| / v(F)$ measures (relative) severity of punishments in $A$ and is called the severity ratio and punishments in $A$ are said to be more severe than $A^{\prime}$ if $r_{A} \geq r_{A^{\prime}}$. Some simple changes of punishments may lead to an increase of severity ratio. For instance consider $\mathbb{A}, \hat{\mathbb{A}} \in \mathbf{A}$, if $v\left(F^{\prime}\right) \leq v(F)$ and $v\left(U^{\prime}\right) \geq v(U)$, then $r_{A} \geq r_{\hat{A}}$.

Whether a change in the severity ratio has any effect on the set of equilibrium strategies of the defendant depends on whether there is uncertainty on how the judge evaluates the evidence. If

\footnotetext{
${ }^{5}$ For any given alternative $\mathbb{A}^{\prime}$, even if $v\left(N^{\prime}\right)>0$ an affine transformation of the defendant's utility would normalize the $\mathrm{v}(\mathrm{N})$ to be zero without changing the equilibrium strategies of the defendant.
} 
there are well defined laws on a subject or there have been many similar cases, then the defendant might not face uncertainty about how the evidence will be evaluated. The no-uncertainty situation is a special case of my model where the defendant is one hundred percent sure that the judge is some specific type. I show that if there is no uncertainty about the type of the judge, then the set of equilibrium disclosures of the defendant are not affected when the severity ratio changes.

Theorem 2 Assume $A 1$ - 3. Let the judge's type be known to be $j$. For all pair $\mathbb{A}, \hat{\mathbb{A}} \in \mathbf{A}$, if $d^{*}$ is an equilibrium disclosure strategy for $\Gamma(\Theta, j, \mathbb{A})$, then it is also an equilibrium strategy for $\Gamma(\Theta, j, \hat{\mathbb{A}})$.

The formal proof of the theorem is given in the appendix. The intuition behind the proof can be best given by a graphical example in which I will focus only on the set of threshold equilibria.

Example 1. Suppose the defendant knows that the judge's type is $j$. In other words, the defendant knows how the judge evaluates the evidence. Let $Q$ be a uniform distribution on $[0,1]$ and let $Q\left(x_{o}\right)=0.2$. Let the judge's evaluation function when evidence is disclosed be

$$
g(j, x)=-(2 x-1)
$$

Function $g$ is shown in Fig. 1 where $x$ is on the horizontal axis. The judge compares his evaluation $g(j, x)$ of evidence $x$ against cut-offs $c_{N}$ and $c_{F}$. For example, if the evaluation is greater than $c_{F}$, then the judge chooses $F$.

Now consider the event that defendant sends $m_{o}$. When the defendant uses a threshold strategy with threshold $\underline{x}$, the expression for judge's expected evaluation (10) is calculated using Bayesian updating of her beliefs according equation (9). I obtain the expected evaluation function of the judge:

$$
E_{\mu_{\underline{x}}}(g(j, x))=\frac{\left(\underline{x}^{2}-\underline{x}\right) 0.8}{0.2+0.8 \underline{x}}
$$

Fig.2 shows $E_{\mu_{\underline{x}}}(g(j, x))$ as a function of threshold $\underline{x}$. In this case, for any threshold the judge's expected evaluation would be between $c_{N}$ and $c_{F}$; therefore, the judge chooses $N$.

In order to find a threshold equilibrium, it suffices to compare $g(j, x)$ and $E_{\mu_{\underline{x}}}(g(j, x))$. For $\underline{\hat{x}}$ to be an equilibrium, type $\underline{\hat{x}}$ should have no incentive to deviate and disclose the evidence. Further, the types above $\underline{\hat{x}}$ should have no incentive to deviate and suppress. Consider a value $\underline{\hat{x}} \in$ $(0,1)$ that invokes the same action on Fig. 1 and Fig.2. Any value above $\underline{\hat{x}}$ would invoke a more favorable action than $\underline{\hat{x}}$ for the defendant on Fig.1. Since $\underline{\hat{x}}$ is indifferent between suppressing and disclosing, at values above $\underline{\hat{x}}$ the defendant does not have incentive to suppress. Therefore such an $\underline{\hat{x}}$ is a threshold equilibrium. The set of values for which the judge's evaluation on both Fig. 1 and Fig. 2 results in the same action are the set of threshold equilibria. 
Figure 1: Evidence Disclosed

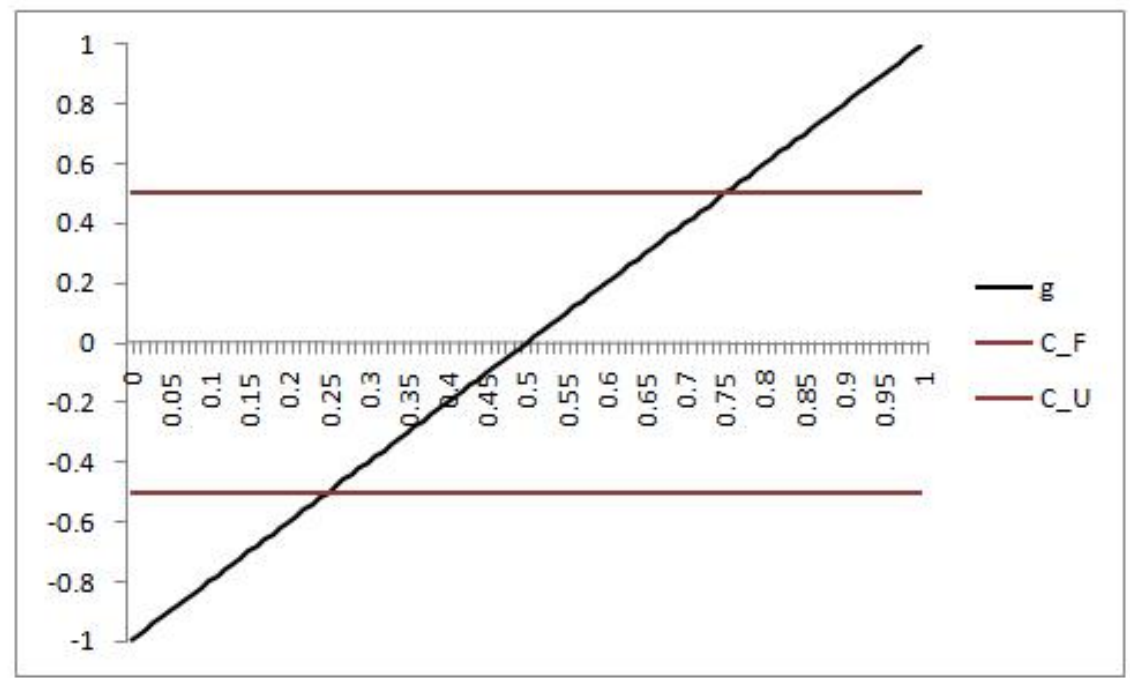

Figure 2: Evidence Suppressed

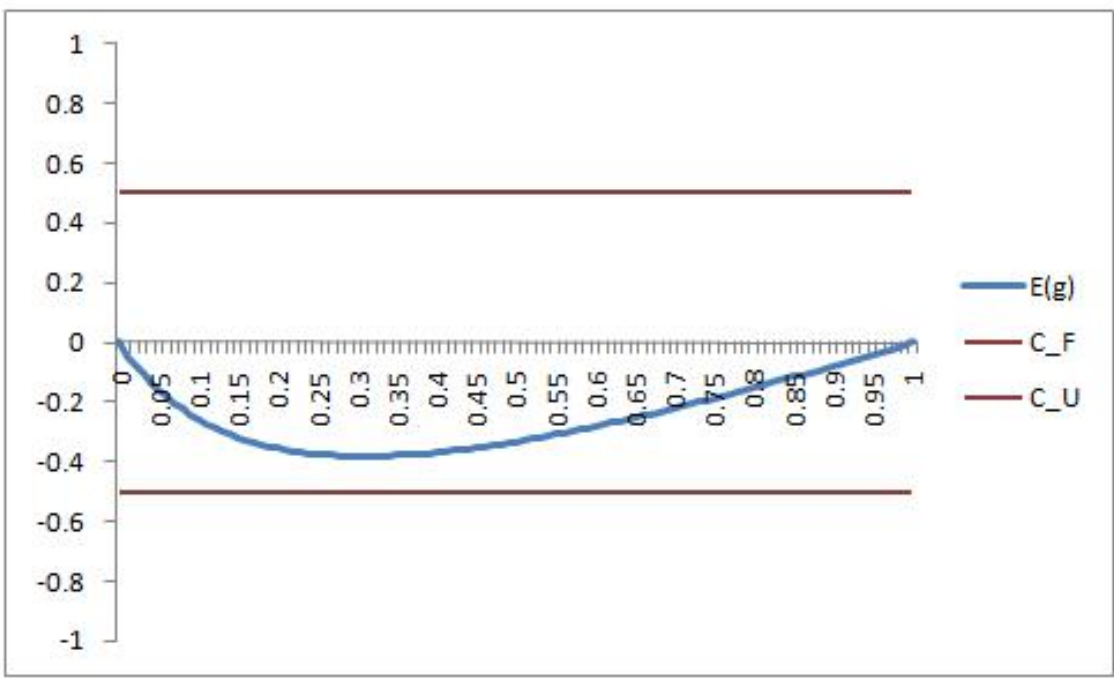


Figure 3: Equilibrium

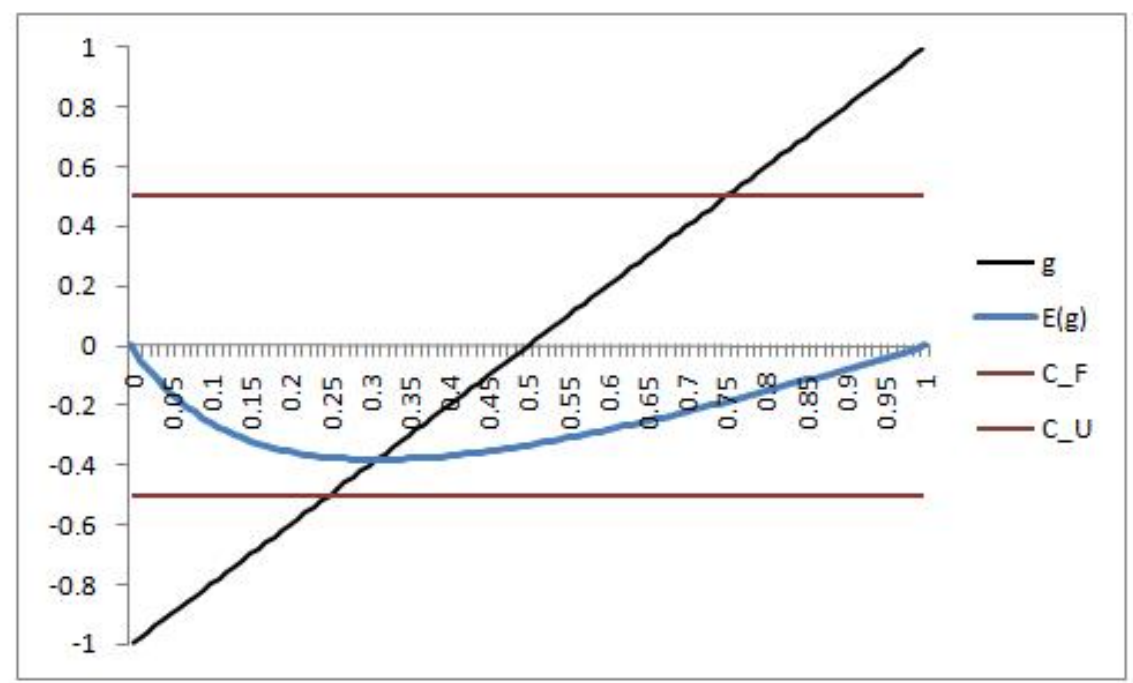

Fig. 3 shows $E_{\mu_{\underline{x}}}(g(j, x))$ and $g(j, x)$ together as functions of $\underline{x}$ and $x$ respectively. The values on the horizontal axis that correspond to the same action on $E_{\mu_{\underline{x}}}(g(j, x))$ and $g(j, x)$ are those that the defendant is indifferent between disclosing the evidence or suppressing it and they constitute the set of threshold equilibria.

Theorem 2 concerns the effect of a change in $\mathbb{A}$ on evidence disclosed in equilibrium. $\mathrm{A}$ change in $\mathbb{A}$ can be expressed as a change in the utility function of the defendant. A threshold value $\underline{x}$ satisfies

$$
\exists y \in \Psi\left(E_{\mu_{\underline{x}}}\right) \text { and } \hat{y} \in \Psi(g(j, x)) \text { such that } y>\tilde{y}
$$

Let the utility function of the defendant be changed from $v$ to some $\hat{v}$ with $\hat{v}(F)>\hat{v}(N)>$ $\hat{v}(U)$. A change from $v$ to $\hat{v}$ is equivalent to a monotone increasing transformation of $y$ and $\tilde{y}$ whenever $y$ and $\tilde{y}$ are results of pure actions of the judge. This would not affect the best response of the defendant. Consider one of $y$ and $\tilde{y}$ is mixed. Since the judge would only mix two adjacent actions a change from $v$ to $\hat{v}$, again, would not affect the best response of the defendant.

The defendant knows the evaluation function of the judge and chooses which message to send to invoke the best action, regardless of how much better this action is compared to next best action. However, if the utility function of the defendant is changed such that ordinal preferences 
of the defendant are affected-for example $\hat{v}(F)>\hat{v}(N)=\hat{v}(U)$-then the set of equilibria would be affected. Fig 3. shows that in this case all values in $[0,0.75]$ would be equilibrium thresholds.

Hence, it suffices to know $v(F)>v(N)>v(U)$ to find the set of equilibria and that is all that is used to find the set of equilibria. This implies that the exact values of $v(F), v(N), v(U)$ are irrelevant. The set of equilibria does not change as long as the ordinal preferences of the defendant stay the same. A change in the severity ratio is a cardinal change and this illustrates Theorem 2.

However, if there is uncertainty about the type of judge, a change in severity ratio may have an effect on the disclosures of the defendant. Theorem 3 below shows that a decrease in severity ratio leads the threshold equilibrium strategies of the defendant to be more informative.

Definition. A threshold strategy $d$ with threshold $\underline{x}$ is said to be at least as informative as the threshold strategy $\hat{d}$ with threshold $\underline{\hat{x}}$ if

$$
\underline{x} \leq \underline{\hat{x}}
$$

Theorem 3 Assume $A 1-3$. Let $r_{A}$ and $r_{\hat{A}}$ be severity ratios for $\mathbb{A}, \hat{\mathbb{A}} \in \mathbf{A}$ respectively. If $r_{A}>$ $r_{\hat{A}}$, then for every equilibrium threshold strategy $d^{*}$ of $\Gamma(\Theta, P, \mathbb{A})$, there exists an equilibrium threshold strategy $d^{* *}$ of game $\Gamma(\Theta, P, \hat{\mathbb{A}})$ that is at least as informative as $d^{*}$.

Theorem 3 shows that a decrease in severity ratio of legal actions results in weakly more informative disclosure strategies of the defendant in equilibrium.

Theorem 4 Assume $A 1-3$. Let $r_{A}$ and $r_{\hat{A}}$ be severity ratios for $\mathbb{A}, \hat{\mathbb{A}} \in \mathbf{A}$ respectively. If $r_{A}<r_{\hat{A}}$, then for every equilibrium threshold strategy $d^{*}$ of $\Gamma(\Theta, P, \mathbb{A})$, there exists an equilibrium threshold strategy $d^{* *}$ of game $\Gamma(\Theta, P, \hat{\mathbb{A}})$ that is weakly less informative than $d^{*}$.

Example 1 illustrates a case in which a decrease in the severity of legal punishment leads to a strictly more informative threshold equilibrium strategy under uncertainty.

Example 2. Suppose that the judge can be one of two types, $\mathbb{J}=\{1,2\}$. Let the first type of the judge be the same as in Example 1. The utility functions of two types of the judge are given by

$$
\begin{gathered}
u_{1}(a, x)=-\left((2 x-1)-b_{a}\right)^{2} \\
u_{2}(a, x)=-\left((2 \sqrt[6]{x}-1)-b_{a}\right)^{2}
\end{gathered}
$$


Figure 4: Lenient Judge

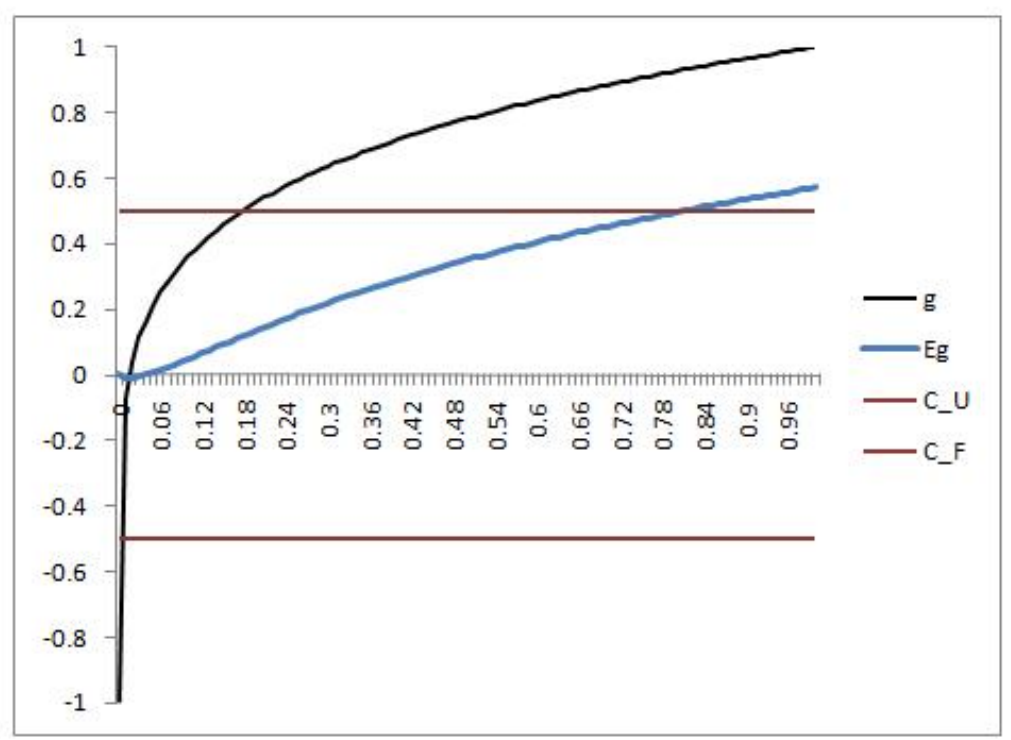

The evaluation functions are given by

$$
\begin{gathered}
g(1, x)=2 x-1 \\
g(2, x)=2 \sqrt[6]{x}-1
\end{gathered}
$$

As seen on Figure 3 and Figure4, $u_{2}(a, x)>u_{1}(a, x)$, for all $x \in(0,1)$ so that the first type's evaluations are always lower than the second judge's evaluations whenever evidence is disclosed. Therefore, the first type is called the strict type and the second type is the lenient type of.

Suppose that both types of judge are equally likely and $Q$ is uniform on $[0,1]$. Moreover let $Q\left(x_{o}\right)=0.2$. The legal standards that the judge uses are given by:

$$
b_{F}=1, b_{N}=0, b_{U}=-1
$$

Fig.3 shows evaluation function $g(1, x)$ and the expected evaluation $E_{\mu_{\underline{x}}}\left(g(1, x) \mid m_{o}\right)$ when the threshold is equal to $x$. The strict type of judge gives $U$ to the defendant whenever there is a disclosure $x$, such that $x<\frac{1}{4}$. As seen in Fig.3, if there is no uncertainty, since $E\left(g(1, x) \mid m_{o}\right)>$ -0.5 for any threshold $\underline{x}<\frac{1}{4}$, the most informative threshold equilibrium is characterized by the threshold $\underline{x}=\frac{1}{4}$.

The second type of judge is lenient and gives $F$ for the values $x>\frac{1}{4}$. Also, since $E\left(g(2, x) \mid m_{o}\right)>$ -0.5 for any threshold the lenient type of the judge gives at least a neutral action when there is no disclosure, as seen in Figure4. 
Let the action set be given as $A=\{U, N, F\}$ and the utility of the defendant be given by $v(F)=1, v(N)=0, v(U)=-100$. Since $v(N)>\frac{1}{2} v(F)+\frac{1}{2} v(U)$, the most informative threshold equilibrium in $\Gamma(\Theta, P, \mathbb{A})$ is characterized by the threshold $\frac{1}{4}$.

Now consider $\mathbb{A}^{\prime}=\left\{U^{\prime}, N^{\prime}, F^{\prime}\right\}$ with $v\left(F^{\prime}\right)=100, v\left(N^{\prime}\right)=0, v\left(U^{\prime}\right)=1$. In that case, since $v(N)>\frac{1}{2} v(F)+\frac{1}{2} v(U)$, the most informative threshold equilibrium strategy is characterized by the threshold $\underline{x}=\frac{729}{4096}<\frac{1}{4}$.

\section{Right to Silence}

The right to silence is adopted in the USA and many other countries to avid self-incrimination. There is no clear evidence that a judge or jury will interpret silence as lack of evidence even under right to silence rule, but it is clear that Bayesian updating might be a strong restriction for an evidence disclosure model. Let's consider the following alternative non-Bayesian updating rule following Che and Severinov (2007):

$$
\hat{E}_{\mu_{\underline{x}}}(g(j, x))=c g\left(j, x_{o}\right)+d \frac{\int_{0}^{\underline{x}} g(j, x) d Q(x)}{c+Q(\underline{x})}
$$

Where $c+d=1$. The non-Bayesian updating rule allows the judge to assign a higher or lower weight to state $x_{o}$ than what the probability distribution on the type space would imply. In other words the judge takes $Q\left(x_{o}\right)=c$ and the only restriction on his beliefs is that the judge uses the same constant for all evidence disclosure strategies.

Equation (16) is equivalent to the Bayesian updating rule when $c=Q\left(x_{o}\right)$ and $d=1 .{ }^{6} \mathrm{Next}$ I show that this generalization does not change any of the previous results.

Theorem 5 Let the judge's beliefs be defined by equation (16), theorems 1-4 hold.

Proof. For any given pair of $c, d$

$$
\hat{E}_{\mu_{\underline{x}}}(g(j, x))=d \frac{\int_{0}^{\underline{x}} g(j, x) d Q(x)}{c+Q(\underline{x})} .
$$

This implies that $\hat{E}_{\mu_{\underline{x}}}(g(j, x))$ is the Bayesian updating rule for a game $\Gamma(\hat{\Theta}, P, A)$ in which $Q\left(\hat{x_{o}}\right)=c$.

\section{Conclusion}

The relationship between incentives to commit legal offense and punishment is studied extensively. This paper studies punishment in another dimension: its relationship with evidence disclosure. In order to study the relationship between magnitude of legal punishment and evidence

\footnotetext{
${ }^{6}$ The beliefs defined by equation (16) are not fully general in the sense that the judge uses the same $c$ and $d$ for any disclosure of the defendant.
} 
disclosure in court measures of severity and informativeness are introduced for the class of strategic evidence disclosure models. The main results are that the equilibrium disclosure of the defendant is not affected by the severity of legal actions when there is no uncertainty on the type of the judge. With uncertainty, however, the defendant can be induced to disclose more information by decreasing severity of legal punishment. Also it is shown that if the severity of the legal system increases, then defendant's equilibrium disclosures becomes less informative. This shows that in a legal system where the laws leave a higher degree of freedom to the judge, a change in the severity ratio of legal actions would affect the evidence disclosed. The results are robust to a class of non-Bayesian beliefs. These results show that it is not sufficient to analyze optimality of legal punishments only from the perspective of incentives to commit crimes, incentives to disclose evidence should be taken to account as well. In fact, the optimal level of punishment is less severe than previous analyzes that do not take evidence disclosure into account would indicate. 


\section{Appendix}

Proof of Theorem 2. Take any pair of SE strategies $\left(f_{j}^{*}, d^{*}\right)$ for game $\Gamma(\Theta, j, \mathbb{A})$, I will show $\left(f^{*}, d^{*}\right)$ are equilibrium strategies also for $\Gamma(\Theta, j, \hat{\mathbb{A}})$. If $d^{*}$ is a best response to $f_{j}^{*}$, this means $d^{*}\left(m^{*} \mid x\right)>0$ implies

$$
\sum_{a} v(a) f_{j}^{*}\left(a \mid m^{*}\right)=\max _{m \in\left\{x, m_{0}\right\}} \sum_{a} v(a) f_{j}^{*}(a \mid m)
$$

Consider an alternative set of actions for the judge, $\hat{\mathbb{A}} \in \mathbf{A}$. If $f_{j}^{*}$ is a mixed strategy, then by (6) there are at most two actions in the support of $f_{j}^{*}$ and if there are two actions in the support, then one of the actions is $N$. Therefore, equation (18) holds if and only if for all pairs $a^{\prime}, a \in \mathbb{A}$ with $v\left(a^{\prime}\right)>v(a)$ either of the following conditions holds:

1. For all $m, f_{j}^{*}\left(a^{\prime} \mid m^{*}\right) \geq f_{j}^{*}\left(a^{\prime} \mid m\right)$ and $f_{j}^{*}\left(a \mid m^{*}\right) \leq f_{j}^{*}(a \mid m)$.

2. If there exists $m \in M_{x}$ such that $f_{j}^{*}\left(a^{\prime} \mid m^{*}\right) \leq f_{j}^{*}\left(a^{\prime} \mid m\right)$, then $f\left(H \mid m^{*}\right)>0$.

The same relationship holds for all pairs $\hat{a}, \hat{a}^{\prime} \in \hat{\mathbb{A}}$. Therefore for every $m^{*}$ such that $d^{*}\left(m^{*} \mid x\right)>0$

$$
\sum_{\hat{a} \in \hat{\mathbb{A}}} v(\hat{a}) f_{j}^{*}\left(\hat{a} \mid m^{*}\right)=\max _{m \in\left\{x, m_{0}\right\}} \sum_{\hat{a} \in \hat{\mathbb{A}}} v(\hat{a}) f_{j}^{*}(\hat{a} \mid m)
$$

Hence, $d^{*}$ and $f^{*}$ are also SE strategies of the game $\Gamma(\Theta, j, \hat{\mathbb{A}})$.

\section{Proof of Theorem 3.}

Take threshold equilibrium strategies $\left(f^{*}, d^{*}\right)$. Let $D_{\emptyset}$ denote the set of types of defendant such that $d^{*}\left(m_{o} \mid x\right)=1$ and let $\underline{x}$ be the highest type of defendant that sends $m_{o}$ under $d^{*}$. For all $\hat{x}$ that is not an element of $D_{\emptyset}$

$$
\sum_{a} \sum_{j} v(a) f_{j}^{*}(a \mid x) P(j) \geq \sum_{a} \sum_{j} v(a) f_{j}^{*}\left(a \mid m_{o}\right) P(j)
$$

Let $\gamma_{x}^{a}=\sum_{j} f_{j}^{*}(a \mid x) P(j)$ and $\gamma_{\emptyset}^{a}=\sum_{j} f_{j}^{*}\left(a \mid m_{o}\right) P(j)$. By (20) defendant discloses $x$ if and only if

$$
\gamma_{x}^{F} v(F)+\gamma_{x}^{U} v(U) \geq \gamma_{\emptyset}^{F} v(F)+\gamma_{\emptyset}^{U} v(U)
$$

Therefore the defendant discloses $x$ if

$$
v(F)\left[\gamma_{x}^{F}-\gamma_{\emptyset}^{F}\right] \geq v(U)\left[\gamma_{\emptyset}^{U}-\gamma_{x}^{U}\right]
$$

This gives for all $x \leq \underline{x}$

$$
v(F)\left[\gamma_{x}^{F}-\gamma_{\emptyset}^{F}\right] \leq v(U)\left[\gamma_{\emptyset}^{U}-\gamma_{x}^{U}\right]
$$


Lemma 1 Let $d^{*}$ be a SE strategy of the defendant and $\underline{x}$ be the threshold under which the defendant sends $m_{o}$. If $x \geq \underline{x}$, then $\gamma_{x}^{F}-\gamma_{\emptyset}^{F}>0$.

Proof. Take an equilibrium characterized by $\underline{x}$. Expected value of $g(x, j)$ conditional upon no disclosure is given by

$$
E\left[g(x, j) \mid m_{o}\right]=\frac{\int_{0}^{\underline{x}} g(x, j) d Q(x)}{Q\left(x_{o}\right)+Q[0, \underline{x}]}
$$

This implies if $E\left[g(x, j) \mid m_{0}\right] \geq c_{F}$ then $g(\underline{x}, j)>c_{F}$. So the set of types of listener that grants $F$ to $x$ is a superset of types that grant $F$ to $m_{o}$.

I want to show that there exists $\underline{\hat{x}} \in[0, \underline{x}]$ such that for all $x \leq \underline{\hat{x}}$, the defendant suppresses the evidence and for all $x>\underline{\hat{x}}$ discloses evidence in game $\Gamma(\Theta, P, \hat{\mathbb{A}})$.

Case 1. Suppose for all $x \leq \underline{x}$

$$
v(\hat{F})\left[\gamma_{x}^{F}-\gamma_{\emptyset}^{F}\right] \leq v(\hat{U})\left[\gamma_{\emptyset}^{U}-\gamma_{x}^{U}\right]
$$

holds. Since $\underline{x}$ is a threshold in $\Gamma(\Theta, P, \mathbb{A})$, for all $x>\underline{x}$ we have

$$
v(F)\left[\gamma_{x}^{F}-\gamma_{\emptyset}^{F}\right] \geq v(U)\left[\gamma_{\emptyset}^{U}-\gamma_{x}^{U}\right]
$$

If $\gamma_{\emptyset}^{U}-\gamma_{x}^{U} \geq 0$, then by Lemma 1 and by the fact that $v(\hat{U})<0$ we have

$$
\forall x>\underline{x}, v(\hat{F})\left[\gamma_{x}^{F}-\gamma_{\emptyset}^{F}\right] \geq v(\hat{U})\left[\gamma_{\emptyset}^{U}-\gamma_{x}^{U}\right]
$$

and therefore incentives to disclose $x$ are not changed. If $\gamma_{\emptyset}^{U}-\gamma_{x}^{U}<0,(21)$ and Lemma 2 implies

$$
\frac{\gamma_{x}^{F}-\gamma_{\emptyset}^{F}}{\left|\gamma_{\emptyset}^{U}-\gamma_{x}^{U}\right|} \geq \frac{|v(U)|}{v(F)}
$$

As severity ratio decreases we have

$$
\frac{\gamma_{x}^{F}-\gamma_{\emptyset}^{F}}{\left|\gamma_{\emptyset}^{U}-\gamma_{x}^{U}\right|} \geq \frac{|v(\hat{U})|}{v(\hat{F})}
$$

Therefore $d^{*}$ is an equilibrium of the $\Gamma(\Theta, P, \hat{\mathbb{A}})$.

Case 2. The nontrivial case is that

$$
v(\hat{F})\left[\gamma_{\underline{x}}^{F}-\gamma_{\emptyset}^{F}\right]>v(\hat{U})\left[\gamma_{\emptyset}^{U}-\gamma_{\underline{x}}^{U}\right]
$$

Consider the correspondence (12) which gives the set of defendant utilities for any given $k$.

$$
\hat{\Psi}(k):=\operatorname{conv}\left\{v(\hat{a}) \mid c_{a_{+}} \geq k \geq c_{a}\right\}
$$


By (23) the set

$$
\sum \hat{\Psi}(g(j, \underline{x})) P(j)-\sum \hat{\Psi}\left(E_{\mu_{\underline{x}}} g(j, x)\right) P(j)
$$

contains a non-negative value. Moreover by $A 5$ the difference

$$
\sum \hat{\Psi}(g(j, 0)) P(j)-\sum \hat{\Psi}\left(E_{\mu_{0}} g(j, x)\right) P(j)
$$

attains a non-positive value. Since the difference correspondence

$$
\sum \hat{\Psi}(g(j, \underline{x})) P(j)-\sum \hat{\Psi}\left(E_{\mu_{\underline{x}}} g(j, x)\right) P(j)
$$

is u.h.c, compact and convex valued there exists a $\underline{\hat{x}} \in[0, \underline{x}]$ such that

$$
0 \in \sum \hat{\Psi}(g(j, \underline{\hat{x}})) P(j)-\sum \hat{\Psi}\left(E_{\mu_{\underline{\hat{x}}}} g(j, \underline{\hat{x}})\right) P(j)
$$

Therefore there exists a weakly more informative equilibrium in $\Gamma(\Theta, P, \hat{\mathbb{A}})$.

Proof of Theorem 4. By Lemma 2 and inequality (22), the defendant $\underline{x}$ chooses to send $m_{o}$ only if

$$
\frac{v(F)}{|v(U)|} \leq \frac{\gamma_{\emptyset}^{U}-\gamma_{\underline{x}}^{U}}{\gamma_{\underline{x}}^{F}-\gamma_{\emptyset}^{F}}
$$

As severity ratio increases

$$
\frac{v(\hat{F})}{|v(\hat{U})|}<\frac{v(F)}{|v(U)|} \leq \frac{\gamma_{\emptyset}^{U}-\gamma_{\underline{x}}^{U}}{\gamma_{\underline{x}}^{F}-\gamma_{\emptyset}^{F}}
$$

This implies that

$$
\sum \hat{\Psi}(g(j, \underline{x})) P(j)-\sum \hat{\Psi}\left(E_{\mu_{\underline{x}}} g(j, x)\right) P(j)
$$

contains a non-positive value. A5 implies that

$$
\sum \hat{\Psi}(g(j, 1)) P(j)-\sum \hat{\Psi}\left(E_{\mu_{1}} g(j, x)\right) P(j)
$$

attains a non-negative value. Therefore there exists $\hat{x} \in[\underline{x}, 1]$ such that

$$
0 \in \sum \hat{\Psi}(g(j, \underline{\hat{x}})) P(j)-\sum \hat{\Psi}\left(E_{\mu_{\underline{x}}} g(j, \underline{\hat{x}})\right) P(j)
$$

We conclude that there exists a weakly less-informative equilibrium in $\Gamma(\Theta, P, \hat{\mathbb{A}})$. 


\section{References}

Becker, G. (1968): “Crime and Punishment: An Economic Approach,” Journal of Political Economy, 76, 169-217.

BULL, J., AND J. WATSON (2004): “Evidence disclosure and verifiability,” Journal of Economic Theory, 118(1), $1-31$.

- (2007): "Hard Evidence and Mechanism Design," Games and Economic Behavior, 58(1), 75-93.

Che, Y.-K., And S. Severinov (2007): “Lawyer Advising in Evidence Disclosure,” Discussion paper, University Library of Munich, Germany.

Daughety, A. F., And J. F. Reinganum (2000): “Appealing Judgments,” RAND Journal of Economics, 31(3), 502-526.

DE Clippel, G. (2008): "An Axiomatization of the Inner Core Using Appropriate Reduced Games," Journal of Mathematical Economics, 44(3-4), 316-323.

Dezhbakhsh, H., P. H. Rubin, and J. M. Shepherd (2003): "Does Capital Punishment Have a Deterrent Effect? New Evidence from Postmoratorium Panel Data," American Law and Economics Review, 5(2), 344-376.

Donohue, J. J., And J. Wolfers (2009): "Estimating the Impact of The Death Penalty on Murder," American Law and Economics Review, 11(2), 249-309.

DYE, R. A. (1986): “Proprietary and Nonproprietary Disclosures," Journal of Business, 59(2), 331-66.

Glazer, J., And A. Rubinstein (2004): “On Optimal Rules of Persuasion,” Econometrica, 72(6), 1715-1736.

- (2006): "A Study in the Pragmatics of Persuasion: A Game Theoretical Approach," Theoretical Economics, 1(4), 395-410.

Milgrom, P. R. (1981): "Good News and Bad News: Representation Theorems and Applications," Bell Journal of Economics, 12(2), 380-391.

Mocan, H. N., And R. K. GitTings (2003): “Getting Off Death Row: Commuted Sentences and the Deterrent Effect of Capital Punishment," Journal of Law \& Economics, 46(2), 453-78. 
Posner, R. A. (1999): “An Economic Approach to the Law of Evidence," Stanford Law Review, 51(6), 1477-1546.

Seidmann, D. J. (2005): “The Effects of a Right to Silence," Review of Economic Studies, 72(2), 593-614.

Seidmann, D. J., And A. STein (2000): "The Right to Silence Helps the Innocent: A GameTheoretic Analysis of the Fifth Amendment Privilege," .

SHER, I. (2008): “Persuasion and Limited Communication,” Discussion paper.

SHIN, H. (1994): “The Burden of Proof in a Game of Persuasion," Journal of Economic Theory, 64(1), 253-264.

SHIN, H. S. (1998): "Adversarial and Inquisitorial Procedures in Arbitration," RAND Journal of Economics, 29(2), 378-405.

Tullock, G. (1974): “Does Punishment Deter Crime,” The Public Interest, 36, 103-111. 